\title{
THE STUDY OF MAGNETO HYDRODYNAMIC FREE CONVECTIVE FLOW AND HEAT TRANSFER IN A POROUS MEDIUM WITH HEAT GENERATION, RADIATION ABSORPTION AND CHEMICAL REACTION
}

\author{
George Buzuzi ${ }^{1 \S}$, Addlight N. Buzuzi ${ }^{2}$, \\ Takura Nyamayaro ${ }^{3}$, William Manamela ${ }^{4}$, \\ Kagiso S. Ramolotja ${ }^{5}$ \\ 1,3,4,5 Department of Mathematics and Physics \\ Cape Peninsula University of Technology \\ P.O. Box 1906, Bellville 7535, SOUTH AFRICA \\ ${ }^{2}$ Department of Further Education and Training \\ Cape Peninsula University of Technology \\ Highbury Rd, Mowbry, 8000, SOUTH AFRICA
}

\begin{abstract}
A study for the problem of unsteady convection flow of a viscous incompressible electrically conducting micro-polar fluid in a porous medium past an inclined moving porous plate in the presence of a transverse magnetic field, heat generation, radiation absorption, chemical reaction and suction is presented. The porous plate moves with a constant velocity and the stream velocity is assumed to follow an exponentially increasing perturbation law. The porous plate absorbs the polar fluid with a suction velocity which varies with time. The dimensionless governing equations are solved analytically by perturbation technique. The numerical results are also compared with corresponding Newtonian fluid flow problems. The effects of various parameters on the velocity, micro-rotation, temperature, concentration fields, skin friction coefficient, couple stress coefficient, Nusselt number and Sherwood number are presented in graphical and tabular form.
\end{abstract}

AMS Subject Classification: 65N06, 65N15, 65M06, 76S05

Key Words: convective flow; MHD; slip flow; inclined plate; porous medium

Revised: July 15, 2020

(c) 2020 Academic Publications

$\S$ Correspondence author 


\section{Introduction}

Polar fluids are fluids consisting of randomly oriented molecules whose fluid elements undergo translational and rotational movements (Roja et al. [15]). The fluid has micro-structure belonging to a class of fluids with asymmetrical stress tensor (Kim [12], Kim and Fedorov [13], Bakr [4]). The behaviour of fluid with suspended particles cannot be explained accurately using the newtonian fluid flow theory but is better explained using the non-newtonian fluid flow theory which embraces polar fluids, hence the recent growing interest in the theory of micro-polar fluids. Physically, micro-polar fluids represents fluid consisting of randomly oriented particles suspended in a viscous medium such as polymer fluids, fluid suspension, human and animal blood, and colloidal fluids (Roja [15], Bakr [4]). The theory of micro-polar fluids was proposed and developed by Eringen [9]. Ahmadi [1] discussed the self-similar solution of incompressible micro-polar boundary layer flow over a semi-infinite plate. Soudalgeker and Takhar [17] on the other hand analysed the flow and heat transfer past a continuously moving plate in a micro-polar fluid and Kim [12] investigated the unsteady MHD convection flow of polar fluid past a vertical moving porous plate in a porous medium. The transient mixed radiative convection flow of a micro-polar fluid past a moving semi-infinite vertical porous plate was studied by Kim and Fedorov [13]. Roja et al. [15] presented a convection-radiation interaction on unsteady MHD flow of a micro-polar fluid over a vertical moving porous plate embedded in a porous medium with heat source and soret effects.

The effect of heat generation or absorption has received a lot of attention due to its importance in fluids undergoing exothermic and endothermic chemical reaction. Hady et al. [10] studied an MHD free convection heat and mass transfer flow past an inclined surface with heat generation. Sharma et al. [16] investigated the effect of magnetic field and heat absorption on unsteady free convection and heat transfer flow in a micro-polar fluid past a semi-infinite moving plate with viscous dissipation. On the other hand Santer Reddy et al. [14] studied the radiation effects on MHD mixed convection flow of a micropolar fluid past a semi-infinite plate in a porous medium with heat generation.

The effect of chemical reaction and radiation absorption on the unsteady MHD free convection Newtonian flow past a semi-infinite permeable moving plate with heat source and suction was investigated by Ibrahim et al. [11]. Deka et al. [8] studied the effect of chemical reaction on an unsteady flow past an infinite vertical plate with constant heat and mass transfer. Chamkha [7] investigated the MHD flow of a uniformly stretched vertical permeable surface in the presence of heat generation/absorption and a chemical reaction. Roja 
et al. [15] studied the effect of chemical reaction and thermal radiation on hydromagnetic free convection heat and mass transfer for a micro-polar fluid past a semi-infinite vertical moving porous plate in the presence of thermal diffusion and heat generation.

In the present work we consider the case of unsteady MHD convection flow of micro-polar fluid past an inclined moving porous medium with varying suction velocity in the presence of a transverse magnetic field, heat generation, radiation absorption and chemical reaction. The study of flow over an inclined plate was studied by a considerable number of researchers. Investigation into the effects of varying suction and thermophoresis on steady MHD flow over a semi-infinite permeable inclined plate in the presence of radiation was done by Alam et al. [2]. In the subsequent year Alam et al. [3] investigated the transient MHD free convective heat and mass transfer flow with thermophoresis past a radiate inclined permeable plate in the presence of temperature dependent viscosity. Uddin [18] did an analysis on steady convective flow of micro-polar fluids along an inclined flat plate with variable electric conductivity and uniform surface heat flux. Buzuzi et al. [5] performed analysis on the effects of various parameters on an MHD flow over an inclined plate using some novel numerical methods. Most recently Buzuzi and Buzuzi [6] conducted a study on unsteady MHD flow past a vertical inclined plate in a porous medium with variable plate temperature with suction in a slip flow regime using perturbation analysis. The problem of unsteady MHD flow of heat generating micro-polar fluid over an inclined surface coupled with heat generation and chemical reaction has received little attention, hence motivation for the current study.

The rest of the paper is organized as follows. In Section 2 we present the model formulation. Section 3 is concerned with the analytical method of solution. Section 4 provides the results and a thorough discussion of the results. Finally, Section 5 presents concluding remarks.

\section{Mathematical analysis}

We consider a two dimensional unsteady flow of a laminar, viscous, incompressible electrically conducting and heat generating /absorbing fluid past a moving inclined permeable plate subjected to a transverse magnetic field in the presence of pressure gradient, radiation absorption and a chemical reaction. Let the $x^{*}$-axis be directed along the porous plate and the $y^{*}$-axis be normal to the plate. The concentration of of species far from the plate is infinitesimally small and so the Soret and Dufour effects are ignored. The assumption that 
the porous plate is semi-infinite means that the flow variables are functions of $y^{*}$ and $t^{*}$ only. A homogenous first order chemical reaction between the fluid and the species concentration is assumed. Then the magnetohydrodynamic unsteady convective boundary layer equations of mass, momentum, energy and concentration read

$$
\begin{aligned}
\frac{\partial v^{*}}{\partial y^{*}} & =0 \\
\frac{\partial u^{*}}{\partial t^{*}}+v^{*} \frac{\partial u^{*}}{\partial y^{*}} & =-\frac{1}{\rho} \frac{\partial p}{\partial x^{*}}+\left(\nu+\nu_{r}\right) \frac{\partial^{2} u^{*}}{\partial y^{* 2}} \\
+g \beta_{t}\left(T-T_{\infty}\right) \cos \psi & +g \beta_{m}\left(C^{*}-C_{\infty}\right) \cos \psi-\frac{\nu u^{*}}{k^{*}} \\
& -\frac{\sigma B_{o}{ }^{2}}{\rho^{*}} u^{*}+2 \nu_{r} \frac{\partial \omega^{*}}{\partial y^{*}}, \\
\rho J^{*}\left(\frac{\partial \omega^{*}}{\partial t^{*}}+\nu^{*} \frac{\partial \omega^{*}}{\partial y^{*}}\right) & =\gamma \frac{\partial^{2} \omega^{*}}{\partial y^{*, 2}}, \\
\frac{\partial T}{\partial t^{*}}+v^{*} \frac{\partial T}{\partial y^{*}}=\alpha \frac{\partial^{2} T}{\partial y^{* 2}} & +Q_{1}^{*}\left(C^{*}-C_{\infty}\right)-Q_{2}\left(T-T_{\infty}\right), \\
\frac{\partial C^{*}}{\partial t^{*}}+v^{*} \frac{\partial C^{*}}{\partial y^{*}} & =D \frac{\partial^{2} C^{*}}{\partial y^{* 2}}-k^{*}{ }_{1}\left(C^{*}-C_{\infty}\right),
\end{aligned}
$$

where $u^{*}$ and $v^{*}$ are the components of dimensional velocities along the $x^{*}$ and $y^{*}$ directions respectively, $\rho$ is the fluid density, and $\nu$ the kinematic viscosity, $\nu_{r}$ the kinematic rotational viscosity, $\beta_{t}$ the coefficient of thermal expansion of fluid, and $\beta_{m}$ is the coefficient of concentration expansion, $k^{*}$ the permeability of the porous medium, $\sigma$ is the electrical conductivity of the fluid, $B_{o}$ is the magnetic induction, $\omega^{*}$ is the component of the angular velocity vector normal to the $x y$-plane, $\alpha$ is the effective fluid thermal diffusivity, $\psi$ is plate angle of inclination, $J^{*}$ the micro-inertia density, $\gamma$ the spin gradient viscosity, $T$ is the temperature of the fluid in the boundary layer, $C^{*}$ is the concentration of fluid in the boundary layer, $Q_{1}^{*}$ is the coefficient of proportionality of the absorption of radiation, $g$ is gravitational acceleration, $k_{1}^{*}$ is the chemical reaction parameter, $D$ is the coefficient of molecular diffusivity, $Q_{2}$ is the dimensional heat absorption coefficient.

The porous plate is assumed to move with constant velocity $u_{p}^{*}$ directed upwards in the $x^{*}$ direction. It is also assumed that the velocities $u^{*}$ and $v^{*}$, temperature $T$, and concentration $C^{*}$ vary exponentially with time. Given the above assumptions, the appropriate boundary conditions for the velocity, 
microrotation, temperature and concentration fields are

$$
\begin{aligned}
u^{*}=u_{p}^{*}, T^{*} & =T_{w}+\epsilon\left(T_{w}-T_{\infty}\right) e^{n^{*} t^{*}}, C^{*}=C_{w}+\epsilon\left(C_{w}-C_{\infty}\right) e^{n^{*} t^{*}}, \\
\frac{\partial \omega^{*}}{\partial y^{*}} & =-\frac{\partial^{2} u^{*}}{\partial y^{* 2}} \text { at } y^{*}=0, \\
u^{*} \rightarrow U_{\infty}^{*} & =U_{0}\left(1+\epsilon e^{n^{*} t^{*}}\right), T \rightarrow T_{\infty}, C \rightarrow C_{\infty}, \omega^{*} \rightarrow 0 \\
& \text { as } y^{*} \rightarrow \infty,
\end{aligned}
$$

where $C_{w}$ and $T_{w}$ are wall dimensional concentration and temperature respectively. $C_{\infty}$ and $T_{\infty}$ are the free stream dimensional concentration and temperature respectively and $U_{o}$ and $n^{*}$ are the scale of free stream velocity and a scalar constant respectively. From the continuity equation (1) it is deduced that the suction velocity is a function of time only and therefore we assume that it takes the following form:

$$
V^{*}=-V_{0}\left(1+\epsilon A e^{n^{*} t^{*}}\right),
$$

where $V_{0}$ is the scale of function velocity and $A$ and $\epsilon$ are small such that $\epsilon<<1$ and $\epsilon A<<1$. Outside the boundary layer, equation 2 gives

$$
-\frac{1}{\rho} \frac{d p^{*}}{d x^{*}}=\frac{d U_{\infty}^{*}}{d t^{*}}+\frac{\nu}{k^{*}} U_{\infty}^{*}+\frac{\sigma}{\rho} B_{o}^{2} U_{\infty}^{*} .
$$

We introduce the following dimensionless quantities:

$$
\begin{aligned}
& y=\frac{V_{0} y^{*}}{v}, t=\frac{V_{0}^{2} t^{*}}{v}, n=\frac{n^{*} \nu}{V_{0}^{2}}, \nu=\frac{\mu}{\rho}, J=\frac{V_{0}^{2} J^{*}}{\nu^{2}}, \\
& \theta=\frac{T-T_{\infty}}{T_{W}-T_{\infty}}, \phi=\frac{C^{*}-C_{\infty}}{C_{w}-C_{\infty}}, u=\frac{u^{*}}{U_{0}}, P_{r}=\frac{\nu}{\alpha}, \\
& u_{p}=\frac{u_{p}^{*}}{U_{0}}, U_{\infty}=\frac{U_{\infty}^{*}}{U_{0}}, \omega=\frac{\nu}{U_{0} V_{0}} \omega^{*}, k=\frac{k^{*} V_{0}^{2}}{\nu^{2}}, S_{c}=\frac{\nu}{D}, \\
& G_{m}=\nu \beta_{m} g \frac{\left(C_{w}-C_{\infty}\right) \cos \psi}{U_{0} V_{0}^{2}}, G_{T}=\nu \beta_{t} g \frac{\left(T_{w}-T_{\infty}\right) \cos \psi}{U_{0} V_{0}^{2}}, \\
& M=\frac{\sigma \nu B_{0}^{2}}{\rho V_{0}^{2}}, Q_{2}=\frac{Q_{2}^{*} \nu}{\rho c_{p} V_{0}^{2}}, Q_{1}=\frac{\nu Q_{1}^{*}\left(C_{w}-C_{\infty}\right)}{\left(T_{w}-T_{\infty}\right) V_{0}^{2}}, \\
& k_{1}=\frac{k_{1}{ }^{*} \nu}{V_{0}^{2}}, \gamma=\mu J^{*}\left(1+\frac{\beta}{2}\right), \beta=\frac{\Lambda}{\mu}=\frac{\nu_{r} \nu}{V_{0}}, N=\frac{1}{k},
\end{aligned}
$$


where $P_{r}$ is the Prandtl number, $G_{m}$ is the mass Grashof number, $G_{T}$ is the thermal Grashof number, $S_{c}$ is the Schmidt number, $\Lambda$ is the coefficient of gyroviscosity ratio, $M$ is the magnetic field parameter and $Q_{1}$ is the absorption of radiation parameter, $k_{1}$ is the chemical reaction parameter, $Q_{2}$ is the heat source parameter, $\beta$ is the dimensional viscosity ratio and $\mu$ is the viscosity of fluid. In view of equations (8)-(9) the non-dimensional form the governing equations (1)-(5) read:

$$
\begin{gathered}
\frac{\partial u}{\partial t}-\left(1+\epsilon A e^{n t}\right) \frac{\partial u}{\partial y}=\frac{\partial U_{\infty}}{\partial t}+(1+\beta) \frac{\partial^{2} u}{\partial y^{2}}+\left(G_{T} \theta+G_{m} \phi\right) \cos \psi \\
+N\left(U_{\infty}-u\right)+2 \beta \frac{\partial \omega}{\partial y} \\
\frac{\partial \omega}{\partial t}-\left(1+\epsilon A e^{n t}\right) \frac{\partial \omega}{\partial y}=\frac{1}{\eta} \frac{\partial^{2} \omega}{\partial y^{2}} \\
\frac{\partial \theta}{\partial t}-\left(1+\epsilon A e^{n t}\right) \frac{\partial \theta}{\partial y}=\frac{1}{P_{r}} \frac{\partial^{2} \theta}{\partial y^{2}}+Q_{1} \phi-Q_{2} \theta \\
\frac{\partial \phi}{\partial t}-\left(1+\epsilon A e^{n t}\right) \frac{\partial \phi}{\partial y}=\frac{1}{S c} \frac{\partial^{2} \phi}{\partial y^{2}}-k_{1} \phi
\end{gathered}
$$

and the the associated boundary conditions (7) is given by the following dimensionless form:

$$
\begin{aligned}
& u=u_{p}, \quad \frac{\partial \omega}{\partial y}=-\frac{\partial^{2} u}{\partial y^{2}}, \quad \theta=1+\epsilon e^{n t}, \quad \phi=1+\epsilon e^{n t} \text { at } y=0, \\
& u=U_{\infty} \rightarrow 1+\epsilon e^{n t}, \quad \theta \rightarrow 0, \quad \omega \rightarrow 0, \quad \phi \rightarrow 0, \quad \text { as } \quad y \rightarrow \infty .
\end{aligned}
$$

\section{Analytical method of solution}

The basic governing equations (11)-(14) are PDE's that cannot be solved in closed form. In order to solve them analytically we reduce the system of PDE's to a system of ODE's in dimensionless form by representing the linear velocity, 
angular velocity, temperature and concentration as

$$
\begin{aligned}
& u(y, t)=u_{0}(y)+\epsilon e^{n t} u_{1}(y)+O\left(\epsilon^{2}\right)+\ldots, \\
& \omega=\omega_{0}(y)+\epsilon e^{n t} \omega_{1}(y)+O\left(\epsilon^{2}\right)+\ldots, \\
& \theta(y, t)=\theta_{0}(y)+\epsilon e^{n t} \theta_{1}(y)+O\left(\epsilon^{2}\right)+\ldots, \\
& \phi(y, t)=\phi_{0}(y)+\epsilon e^{n t} \phi_{1}(y)+O\left(\epsilon^{2}\right)+\ldots
\end{aligned}
$$

Substituting (16) in (11)-(14), and equating the harmonic and non-harmonic terms ignoring terms of $O\left(\epsilon^{2}\right)$ gives the following equations for $\left(u_{0}, \omega_{0}, \theta_{0}, \phi_{0}\right)$ and $\left(u_{1}, \omega 1, \theta_{1}, \phi_{1}\right)$,

$$
\begin{gathered}
(1+\beta) u_{0}^{\prime \prime}+u_{0}^{\prime}-N u_{0}=-G_{T} \theta_{1} \cos \psi-G_{m} \phi_{1} \cos \psi \\
-N-2 \beta \omega_{0}^{\prime}, \\
\omega_{0}^{\prime \prime}+\eta \omega_{0}^{\prime}=0, \\
\theta_{0}^{\prime \prime}+P_{r} \theta_{0}^{\prime}-P_{r} Q_{2} \theta_{0}=-P_{r} Q_{1} \phi_{0}, \\
\phi_{0}^{\prime \prime}+S_{c} \phi_{0}^{\prime}-k_{1} S_{c} \phi_{0}=0,
\end{gathered}
$$

subject to the boundary conditions

$$
\begin{aligned}
& u_{0}=u_{p}, \quad \omega_{0}^{\prime}=-u_{0}^{\prime \prime}, \quad \theta_{0}=1, \quad \phi_{0}=1 \text { on } y=0, \\
& u_{0}=1, \quad \omega_{0} \rightarrow 0, \quad \theta_{0} \rightarrow 0, \quad \phi_{0} \rightarrow 0 \quad \text { as } y \rightarrow \infty,
\end{aligned}
$$

and

$$
\begin{gathered}
(1+\beta) u_{1}^{\prime \prime}+u_{1}^{\prime}-(N+n) u_{1}=-(N+n)-A u_{0}^{\prime} \\
-\left(G_{T} \theta_{1}+G_{m} \phi_{1}\right) \cos \psi-2 \beta \omega_{1}^{\prime}, \\
\omega_{1}^{\prime \prime}+\eta \omega_{1}^{\prime}-n \eta \omega_{1}=-A \eta \omega_{0}^{\prime}, \\
\theta_{1}^{\prime \prime}+P_{r} \theta_{1}^{\prime}-P_{r}\left(Q_{2}+n\right) \theta_{1}=-P_{r} A \theta_{0}^{\prime}-P_{r} Q_{1} \phi_{1}, \\
\phi_{1}^{\prime \prime}+S_{c} \phi_{1}^{\prime}-\left(k_{1}+n\right) S_{c} \phi_{1}^{\prime}=A S_{c} \phi_{0}^{\prime},
\end{gathered}
$$

subject to the boundary conditions

$$
\begin{aligned}
& u_{1}=0, \quad \omega_{1}^{\prime}=-u_{1}^{\prime \prime}, \quad \theta_{1}=1, \quad \phi_{1}=1 \quad \text { on } y=0, \\
& u_{1}=1, \quad \omega_{1} \rightarrow 0, \quad \theta_{1} \rightarrow 0, \quad \phi_{1} \rightarrow 0 \quad \text { as } y \rightarrow \infty,
\end{aligned}
$$


where the prime denotes ordinary differentiation with respect to $y$.

The solution of the equations (17)-(20) and (22)-(25) subject to the boundary conditions (21) and (26) are:

$$
\begin{aligned}
& u_{0}=E e^{-h 7 y}+a_{1} e^{-t_{1} y}+b_{1} e^{-t_{2} y}+c_{1} e^{-h_{3} y}+d_{1} e^{-h_{5} y}+f_{1} e^{-\eta y}+1, \\
& u_{1}=l_{5} e^{-h_{9} y}+d_{0} e^{-t_{1} y}+d_{2} e^{-t_{2} y}+d_{3} e^{-h_{3} y}+d_{4} e^{-h_{1} y}+d_{5} e^{-h_{5} y} \\
& +d_{7} e^{-h_{7} y}+d_{8} e^{-\eta y}+1 \text {, } \\
& \omega_{0}=b e^{-\eta y}, \\
& \omega_{1}=l_{0} e^{-h_{1} y}-k_{m} e^{-\eta y}, \\
& \theta_{0}=\left(1-k_{a}\right) e^{-t_{1} y}+k_{a} e^{-h_{3} y}, \\
& \theta_{1}=E_{3} e^{-t_{2} y}+E_{5} e^{-t_{1} y}+E_{6} e^{-h_{3} y}+E_{7} e^{-h_{5} y}, \\
& \phi_{0}=e^{-h_{3} y}, \\
& \phi_{1}=\left(1-k^{*}\right) e^{-h_{5} y}+k^{*} e^{-h_{3} y},
\end{aligned}
$$

where,

$$
\begin{aligned}
& h_{1}=\frac{\eta+\sqrt{\eta^{2}+4 n \eta}}{2}, \quad h_{3}=\frac{S_{c}}{2}\left(1+\sqrt{1+\frac{4 k_{1}}{S_{c}}}\right) \\
& h_{5}=\frac{S_{c}}{2}\left(1+\sqrt{1+\frac{4\left(k_{1}+n\right)}{S_{c}}}\right), k^{*}=\frac{-A S_{c} h_{3}}{h_{3}^{2}-h_{3} S_{c}-\left(k_{1}+n\right) S_{c}}, \\
& t_{1}=\frac{P_{r}}{2}\left(1+\sqrt{1+\frac{4 Q_{2}}{P_{r}}}\right), t_{2}=\frac{P_{r}}{2}\left(1+\sqrt{1+\frac{4\left(Q_{2}+n\right)}{P_{r}}}\right) \\
& k_{a}=\frac{-P_{r} Q_{1}}{h_{3}^{2}-P_{r} h_{3}-P_{r} Q_{2}}, E_{5}=\frac{P_{r} A t_{1}\left(1-k_{a}\right)}{t_{1}^{2}-P_{r} t_{1}-P_{r}\left(Q_{2}+n\right)}, \\
& E_{6}=\frac{P_{r} A h_{3} k_{a}-P_{r} Q_{1} k^{*}}{h_{3}^{2}-P_{r} h_{3}-P_{r}\left(Q_{2}+n\right)}, E_{7}=\frac{-P_{r} Q_{1}\left(1-k^{*}\right)}{h_{5}^{2}-P_{r} h_{5}-P_{r}\left(Q_{2}+n\right)},
\end{aligned}
$$




$$
\begin{aligned}
& h_{7}=\frac{1}{2(1+\beta)}(1+\sqrt{1+4 N(1+\beta)}), f_{1}=\frac{2 \beta b \eta}{(1+\beta) \eta^{2}-\eta-N} \\
& A_{1}=-\left[G_{m}\left(1-k^{*}\right)+G_{T} E_{7}\right] \cos \psi, b_{1}=\frac{-G_{T} E_{3} \cos \psi}{(1+\beta) t_{2}^{2}-t_{2}-N} \\
& B_{1}=A c_{1} h_{3}-\left(G_{T} E_{6}+G_{m} k^{*}\right) \cos \psi, c_{1}=\frac{-\left(G_{m} k^{*}+G_{T} E_{6}\right) \cos \psi}{(1+\beta) h_{3}^{2}-h_{3}-N}, \\
& d_{0}=\frac{A a_{1} t_{1}-G_{t} E_{5} \cos (\psi)}{(1+\beta) t_{1}^{2}-t_{1}-(N+n)}, d_{1}=\frac{A_{1}}{(1+\beta) h_{5}^{2}-h_{5}-N}, \\
& d_{2}=\frac{A b_{1} t_{2}-G_{T} E_{3} \cos \psi}{(1+\beta) t_{2}^{2}-t_{2}-(N+n)}, d_{3}=\frac{B_{1}}{(1+\beta) h_{3}^{2}-h_{3}-(N+n)}, \\
& d_{5}=\frac{A d_{1} h_{5}-\left(G_{T} E_{7}+G_{m}\left(1-k^{*}\right)\right) \cos \psi}{(1+\beta) h_{5}^{2}-h_{5}-(N+n)}, \quad k_{m}=\frac{-b A \eta}{n}, \\
& d_{7}=\frac{A h_{7} E}{(1+\beta) h_{7}^{2}-h_{7}-(N+n)}, \quad d_{8}=\frac{A * f_{1} \eta-2 \beta \eta k_{m}}{(1+\beta) \eta^{2}-\eta-(N+n)}, \\
& h_{9}=\frac{1}{2(1+\beta)}(1+\sqrt{1+4(1+\beta)(N+n)}) \text {, } \\
& E=\left(u_{p}-1-a_{1}-b_{1}-c_{1}-d_{1}-f_{1}\right) \\
& l_{5}=-\left(1+d_{0}+d_{2}+d_{3}+d_{4}+d_{5}+d_{7}+d_{8}\right), \\
& E_{10}=\left(t_{1}^{2}-h_{9}^{2}\right) d_{0}+\left(t_{2}^{2}-h_{9}^{2}\right) d_{2}+\left(h_{3}^{2}-h_{9}^{2}\right) d_{3}+\left(h_{5}^{2}-h_{9}^{2}\right) d_{5} \\
& +\left(h_{7}^{2}-h_{9}^{2}\right) d_{7}+\left(\eta^{2}-h_{9}^{2}\right) d_{8}, \\
& A_{0}=\left(\left(u_{p}-1\right) h_{7}^{2}+\left(t_{1}^{2}-h_{7}^{2}\right) a_{1}+\left(t_{2}^{2}-h_{7}^{2}\right) b_{1}+\left(h_{3}^{2}-h_{7}^{2}\right) c_{1}\right. \\
& \left.+\left(h_{5}^{2}-h_{7}^{2}\right) d_{1}\right) \text {, } \\
& b=\frac{A_{0}}{\left((1-\beta) \eta^{2}-\eta-N+2 \beta h_{7}^{2}\right)} \times\left((1+\beta) \eta-1-\frac{N}{n}\right) \\
& d_{4}=2 \beta\left(\frac{\frac{A \eta^{2} b}{n}+E_{10}-h_{9}^{2}}{(1-\beta) h_{1}^{2}-h_{1}+2 \beta h_{9}^{2}-(N+n)}\right),
\end{aligned}
$$




$$
\begin{array}{r}
l_{0}=\left(\frac{\frac{A \eta^{2} b}{n}+E_{10}-h_{9}^{2}}{(1-\beta) h_{1}^{2}-h_{1}+2 \beta h_{9}^{2}-(N+n)}\right) \\
\times\left((1+\beta) h_{1}-1-\frac{N+n}{h_{1}}\right) .
\end{array}
$$

By considering (16) the final form of the velocity, microrotation, temperature and concentration distributions in the boundary layer are:

$$
\begin{gathered}
u(y, t)=E e^{-h 7 y}+a_{1} e^{-t_{1} y}+b_{1} e^{-t_{2} y}+c_{1} e^{-h_{3} y}+d_{1} e^{-h_{5} y} \\
+f_{1} e^{-\eta y}+1+\epsilon e^{n t}\left(l_{5} e^{-h_{9} y}+d_{0} e^{-t_{1} y}+d_{2} e^{-t_{2} y}\right. \\
\left.+d_{3} e^{-h_{3} y}+d_{4} e^{-h_{1} y}+d_{5} e^{-h_{5} y}+d_{7} e^{-h_{7} y}+d_{8} e^{-\eta y}+1\right) \\
\theta(y, t)=\left(1-k_{a}\right) e^{-t_{1} y}+k_{a} e^{-h_{3} y}+\epsilon e^{n t}\left(E_{3} e^{-t_{2} y}+E_{5} e^{-t_{1} y}\right. \\
\left.+E_{6} e^{-h_{3} y}+E_{7} e^{-h_{5} y}\right) \\
\omega(y, t)=b e^{-\eta y}+\epsilon e^{n t}\left(l_{0} e^{-h_{1} y}-k_{m} e^{-\eta y}\right) \\
\phi(y, t)=e^{-h_{3} y}+\epsilon e^{n t}\left(\left(1-k^{*}\right) e^{-h_{5} y}+k^{*} e^{-h_{3} y}\right)
\end{gathered}
$$

The skin friction coefficient $C_{f}$, the Sherwood number $S h$, Nusselt number $N u$ and the couple stress coefficient $C_{m}$ at the wall of the plate are defied as follows:

$$
\begin{gathered}
C_{f}=-\left[E h_{7}+a_{1} t_{1}+b_{1} t_{2}+c_{1} h_{3}+d_{1} h_{5}+f_{1} \eta+\epsilon e^{n t}\left(l_{5} h_{9}\right.\right. \\
\left.\left.+d_{0} t_{1}+d_{2} t_{2}+d_{4} h_{1}+{ }_{3} h_{3}+d_{8} \eta+d_{5} h_{5}+d_{7} h_{7}\right)\right] \\
\frac{S h}{R e}=\left.\frac{\partial \phi}{\partial y}\right|_{y=0}=h_{3}+\epsilon e^{n t}\left[h_{5}\left(1-k^{*}\right)+k^{*} h_{3}\right], \\
\frac{N u}{R e}=\left.\frac{\partial \theta}{\partial y}\right|_{y=0}=t_{1}\left(1-k_{a}\right)+h_{3} k_{a}+\epsilon e^{n t}\left(E_{3} t_{2}+E_{5} t_{1}+h_{3} E_{6}+h_{5} E_{7}\right), \\
C_{m}=\left(1+\frac{\beta}{2}\right) \omega^{\prime}(0)=\left(1+\frac{\beta}{2}\right)\left[-b \eta+\epsilon e^{n t}\left(\frac{A b \eta^{2}}{n}-l_{0} h_{1}\right)\right]
\end{gathered}
$$

where $R e$ is the local Reynolds number. 


\section{Results and discussion}

In the current study we have analyzed MHD convective flow of micro-polar heat generating/absorbing fluid over a vertical semi infinite moving permeable plate embedded in a porous medium subjected to transverse magnetic field in the presence of radiation absorption and chemical reaction. Final results are computed for various physical parameters which are presented graphically in Figures 1 - 17 and also in tabular form shown in Tables 1 - 6. In plotting Figures 1 - 17 the following flow conditions were fixed unless specified, $\beta=0.2, \quad n=$ $1, \quad t=1, \quad k_{1}=0 ., \quad M=2, \quad A=0.5, \quad Q_{1}=2, \quad Q_{2}=1, \quad \epsilon=0.1, \quad G_{T}=$ $2, \quad G_{m}=1, \quad P_{r}=1, \quad u_{p}=0.5, \quad S_{c}=0.4$.

The effect of various parameters on the velocity profile are depicted in Figures 1 - 7. In particular Figure 1 shows the effect of viscosity ratio on velocity for $n=1, \quad t=1, \quad k_{1}=0.5, \quad M=0, \quad A=0, \quad Q_{1}=0, \quad Q_{2}=0, \quad \epsilon=0, \quad G_{T}=$ $2, \quad G_{m}=0, \quad P_{r}=1, \quad u_{p}=0, \quad S_{c}=0.4$. The parameter values have been chosen so as to match those by Kim [12] whose graphs match perfectly. It is observed that the velocity profile increases with increasing viscosity ratio $\beta$. It is also noticed that when $\beta>0.5$ the velocity profile for polar fluid is lower than that of the Newtonian fluid $(\beta=0)$ and the relationship is reversed for $\beta \leq 0.5$.

The velocity profile against coordinate $y$ for a combination of different values of the thermal Grashof number $G_{T}$ and mass Grashof number $G_{m}$ is depicted in Figure 2. It is noticed that an increase in $G_{T}$ or $G_{m}$ leads to an increase in the velocity distribution. If both $G_{T}$ and $G_{m}$ increase then the increase in the velocity distribution is magnified accordingly. Similarly if $G_{T}$ or $G_{m}$ are negative the velocity distribution decreases. The decrease is more pronounced if both $G_{T}$ and $G_{m}$ are negative. Figure 3 shows that the velocity rises as the absorption of radiation parameter $Q_{1}$ increases. On the other hand Figure 4 shows that the velocity profile decreases as the heat source parameter $Q_{2}$ increases. It is portrayed from Figure 5 that the velocity profile increases as the chemical reaction parameter $k_{1}$ increases. It is also observed from Figure 6 that as the Schmidt number $S_{c}$ increases, the velocity profile increases near the plate and decreases as you move further away from the plate. The observation is reversed for $S_{c}>1.1$. The plate angle of inclination $\psi$ to the vertical direction on the velocity is depicted in Figure 7. It is observed that the velocity is decreased by increasing the angle of inclination. Increasing $\psi$ has the effect of decreasing the buoyancy force since it is multiplied by $\cos \psi$ and so the velocity decreases. The maximum buoyancy force occurs for $\psi=0$ when the plate is vertical and there is no buoyancy effect for $\psi=\pi / 2$ when the plate is horizontal. 
Figures 8 - 11 display profiles of angular velocity for varying parameter values with $u_{p}=0$. As Figure 8 portrays, an increase in either $G_{T}$ or $G_{m}$ results in a fall in the angular velocity. The decrease is more pronounced if $G_{T}$ and $G_{m}$ are both positive. Similarly if either $G_{T}$ or $G_{m}$ are negative the angular velocity profile increases. The increase is enhanced if $G_{T}$ and $G_{m}$ are both negative. As shown in Figure 9 the angular velocity profile for newtonian fluid $(\beta=0)$ is lower than that of micropolar fluid for $\beta \geq 0.7$. When $\beta<0.7$ the angular velocity profile is higher for newtonian fluid compared with micropolar fluid. The critical value for $\beta$ for which $\omega$ changes sign is dependent on the value of the magnetic parameter $M$. For parameter values in Figure 9 the critical value is $\beta=0.7$. Figure 10 shows how the critical values of $\beta$ changes as the value of $M$ changes. It is shown that the critical $\beta$ values increases as the value of $M$ increases. Figure 11 shows that the angle of inclination has an increasing effect on angular velocity profiles.

Figures 12 - 14 display the temperature profiles across the boundary layer for fixed $\beta=0.2, \quad n=1, \quad t=1, \quad k_{1}=0 ., \quad M=2, \quad A=0.5, \quad Q_{1}=$ $2, \quad Q_{2}=1,, \epsilon=0.1, \quad G_{T}=2, \quad G_{m}=1, \quad P_{r}=1, \quad u_{p}=0.5, \quad S_{c}=0.4$, while $k_{1}, Q_{2}$ and $S_{c}$ vary. It is noticed that the temperature falls with increasing chemical reaction parameter $k_{1}$, heat generation /absorption parameter $Q_{2}$ and Schmidt number $S_{c}$.

The variation of concentration profiles for different values of the Schmidt number $S_{c}$, chemical reaction parameter $k_{1}$ and suction $A$ is depicted in Figures 15 - 17. The effect of increasing the Schmidt number $S_{c}$ and chemical reaction parameter $k_{1}$ is to decrease the concentration profile across the boundary layer. It is seen that as the suction parameter $A$ increases the concentration also increase

The effects of magnetic parameter $M$, heat generation/absorption parameter $Q_{2}$, suction parameter $A$, absorption of radiation parameter $Q_{1}$, inclination angle $\psi$ and chemical reaction parameter $k_{1}$ on the skin friction $C_{f}$, Nusselt number $N u$, Couple wall stress $C_{m}$ and Sherwood number $S h$ is depicted in Tables $1-6$. From Table 1 it is observed that the increase in the magnetic parameter has no effect on both the Nusselt number and the Sherwood number. However the skin friction decreases as the magnetic parameter increases. The Couple wall stress decreases as the magnetic parameter increases for $M<6$ and increases as $M$ increases for $M \geq 6$. From Table 2 it is noticed that as the heat generation/absorption parameter increases the skin friction and the Couple wall stress decreases. It is also observed that the Nusselt number increases with increasing heat generation parameter while the Sherwood number remain unchanged as the heat generation/absorption parameter changes. From 


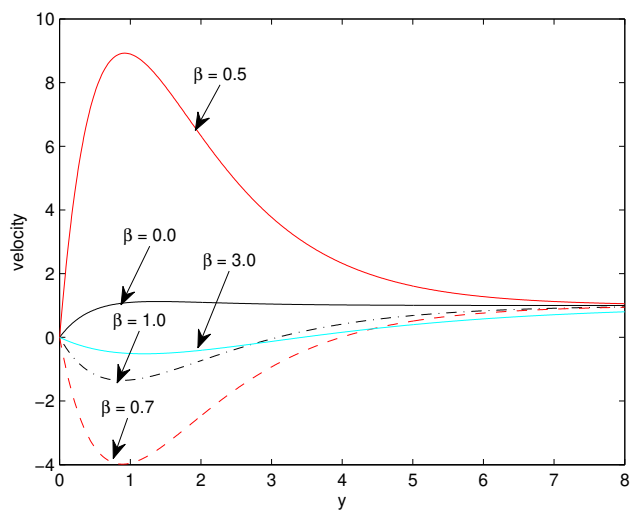

Figure 1: Velocity profile for different values of $\beta$

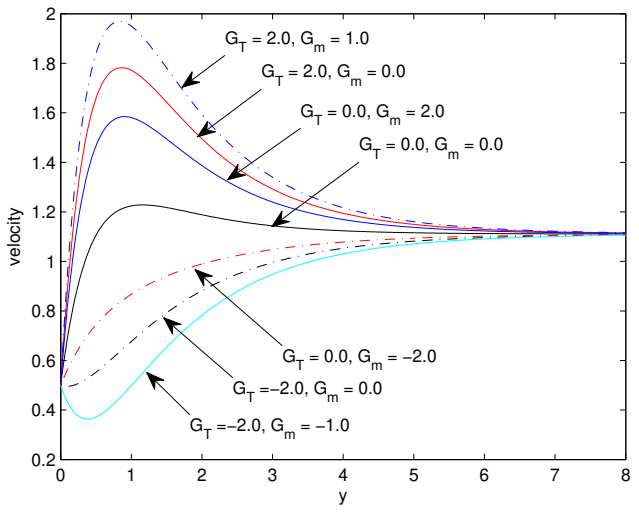

Figure 2: Velocity profile for different values of $Q_{T}$ and $G_{m}$ 


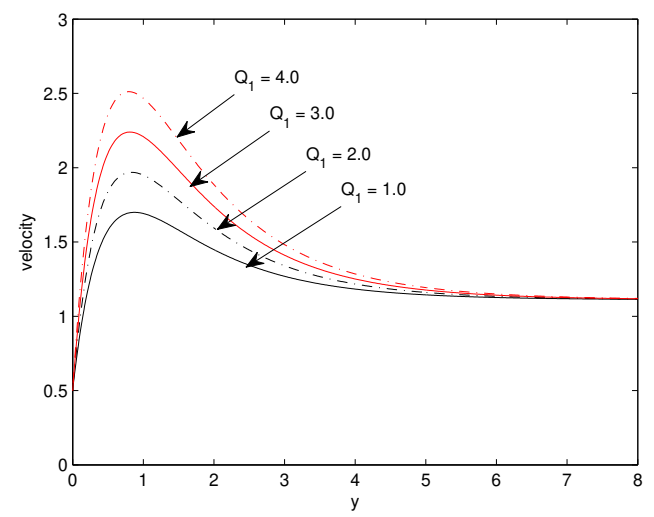

Figure 3: Velocity profile for different values of $Q_{1}$

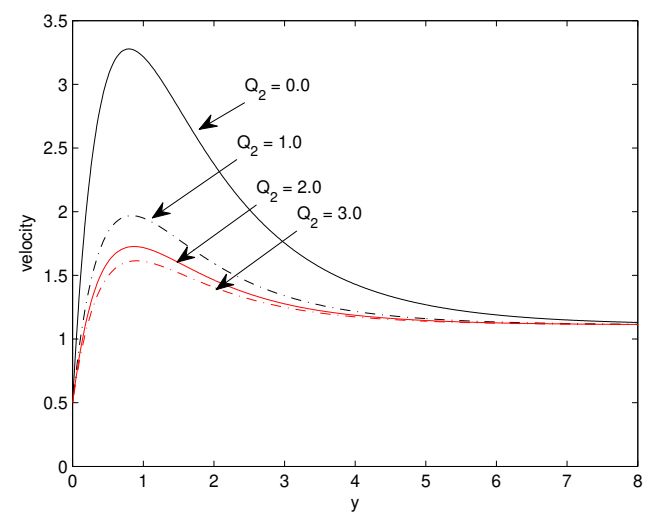

Figure 4: Velocity profile for different values of $Q_{2}$

Tables 3 and 4 it is shown that as $A$ or $Q_{1}$ increases there is an increase in the skin friction and Nusselt number. It is also observed that the Couple wall stress increases with increasing suction parameter and decreases with increasing absorption of radiation parameter. The Sherwood number remains unchanged with variation of $A$ or $Q_{1}$. The effect of the chemical reaction parameter on the skin friction, Nusselt number, Couple wall stress and Sherwood number is displayed in Table 5 . It is noticed that as $k_{1}$ increases there is a decrease in both the skin friction and the Nusselt number while an increase is observed in the 


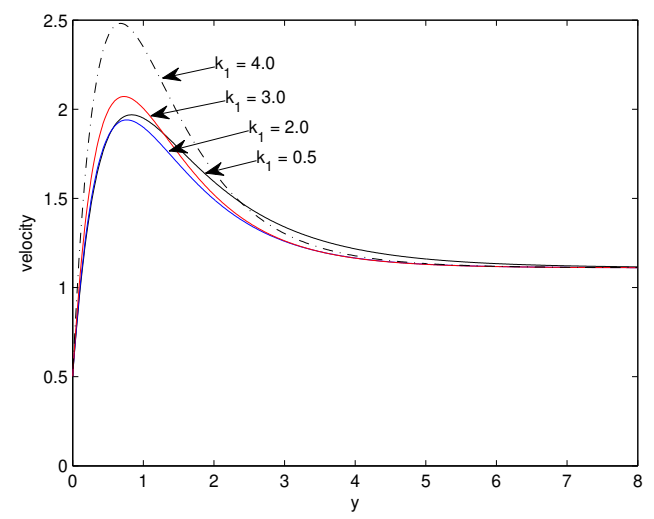

Figure 5: Velocity profile for different values of $k_{1}$

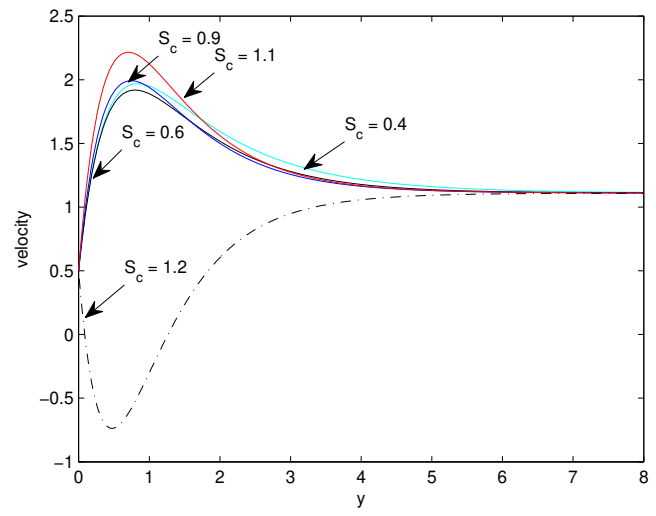

Figure 6: Velocity profile for different values of $S_{c}$

Sherwood number. The Couple wall stress is unchanged with the variation of $k_{1}$. The effect of inclination angle on the skin friction, Nusselt number, Couple wall stress and Sherwood number is depicted in Table 6. It is observed that the inclination angle has a decreasing effect on the skin friction friction and Couple wall stress. However varying the plate inclination angle has no effect on the Nusselt number and Sherwood number. 


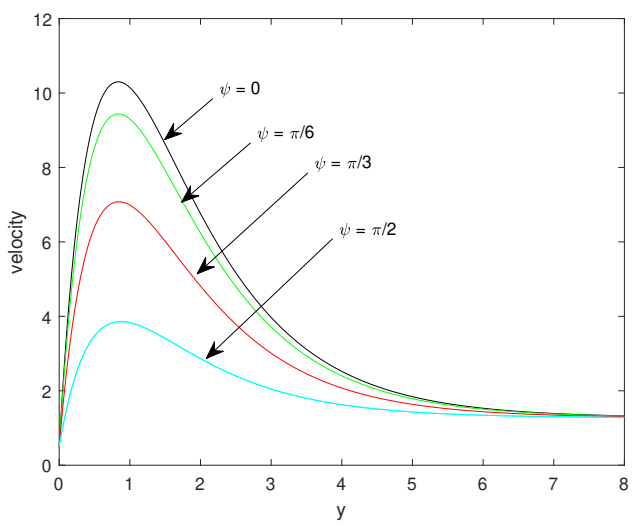

Figure 7: Velocity profile for different values of $\psi$

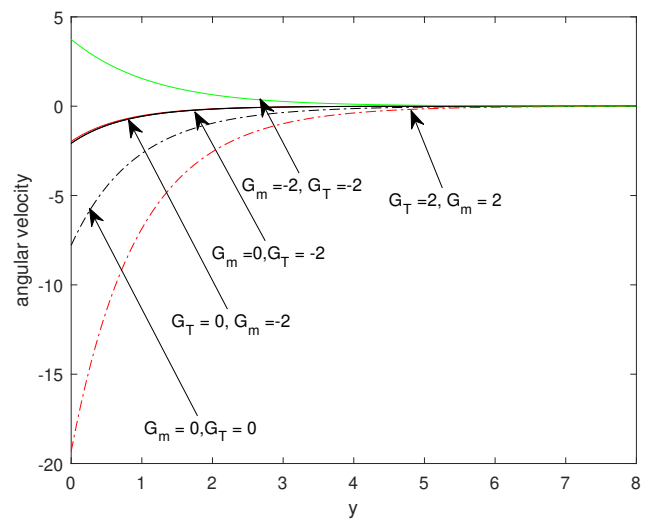

Figure 8: Angular velocity profile for different values of $G_{T}$ and $G_{m}$

\section{Concluding remarks}

In this paper we studied on the effects of heat generation, radiation of absorption, chemical reaction, viscosity, magnetism, plate inclination angle and suction on the unsteady MHD convective flow of micro-polar fluid past a porous medium. The governing equations were transformed into a system of nonlinear differential equations using perturbation analysis. From the numerical study we deduce the following.

1. Velocity increases with increase in the thermal Grashof number, mass Grashof number, absorption of radiation parameter, viscosity ratio, chemical reaction 
Table 1: Effect of $\mathrm{M}$ on skin friction, Nusselt number, Couple wall stress and Sherwood number

\begin{tabular}{ccccc}
\hline $\mathrm{M}$ & $C_{f}$ & $C_{m}$ & $N u R e^{-1}$ & $S h R e^{-1}$ \\
\hline 0.0 & 32.2899 & 93.4927 & 1.0636 & 0.4641 \\
2.0 & 16.1673 & 55.5201 & 1.0636 & 0.4641 \\
5.0 & 12.2484 & 49.5012 & 1.0636 & 0.4641 \\
6.0 & 11.7418 & 49.5204 & 1.0636 & 0.4641 \\
10 & 10.8541 & 52.3838 & 1.0636 & 0.4641 \\
15 & 10.6588 & 58.2806 & 1.0636 & 0.4641 \\
\hline
\end{tabular}

parameter and Schmidt number near the plate.

2. Velocity decreases with increase in the heat generation parameter, inclination angle and Schmidt number further away from the plate.

3. Angular velocity increases with increase in the inclination angle.

4. Angular velocity decreases with increase in the viscosity ratio, thermal Grashof number and mass Grashof number.

5. Temperature falls with increase in the chemical reaction parameter, heat generation parameter and Schmidt number.

6. Concentration decreases with increase in either the chemical reaction parameter or Schmidt number.

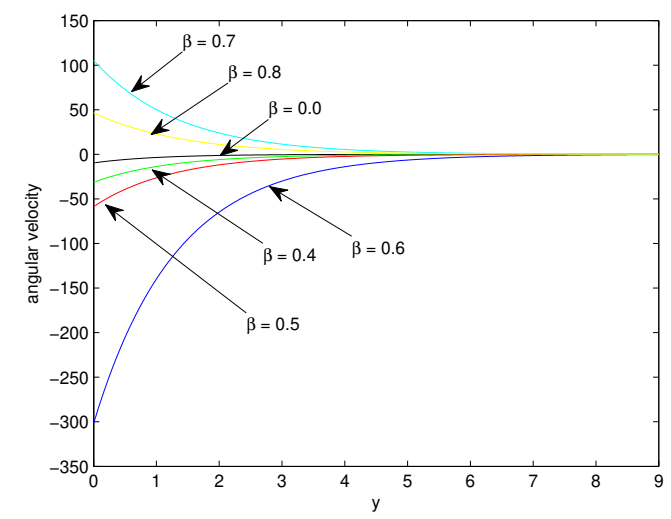

Figure 9: Angular velocity profile for different values of $\beta$ 


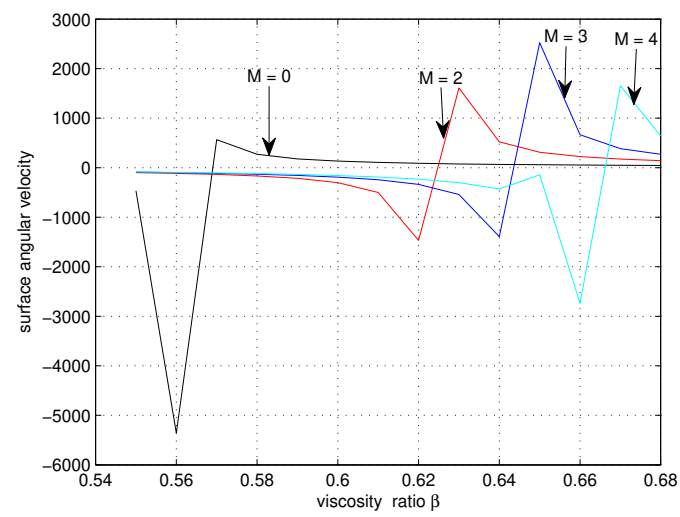

Figure 10: Angular velocity profile for different values of $M$

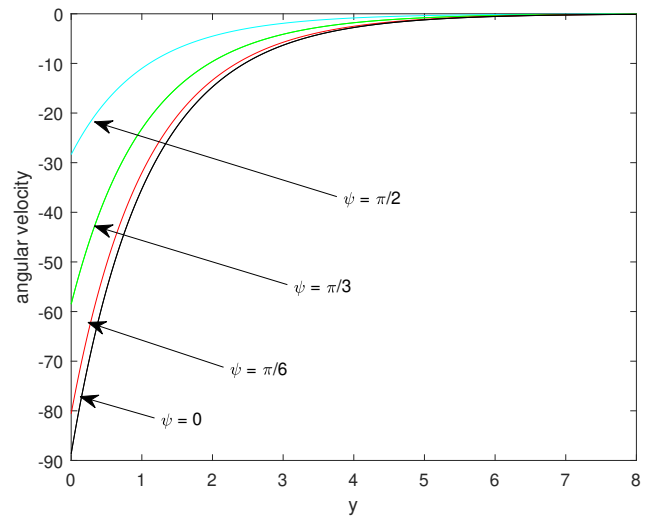

Figure 11: Angular velocity profile for different values of $\psi$

7. Skin friction increases with increase in either the suction parameter or radiation of absorption parameter. On the other hand skin friction decreases with increase in the chemical reaction parameter, magnetic parameter, inclination angle and heat generation parameter.

8. The Nusselt number increases with increase in the suction parameter, radiation of absorption parameter and heat generation parameter. The Nusselt number decreases with an increase in the chemical reaction parameter.

9. Sherwood number increases with increase in the chemical reaction parameter and remain unchanged as $A, Q_{1}$ and $Q_{2}$ vary. 


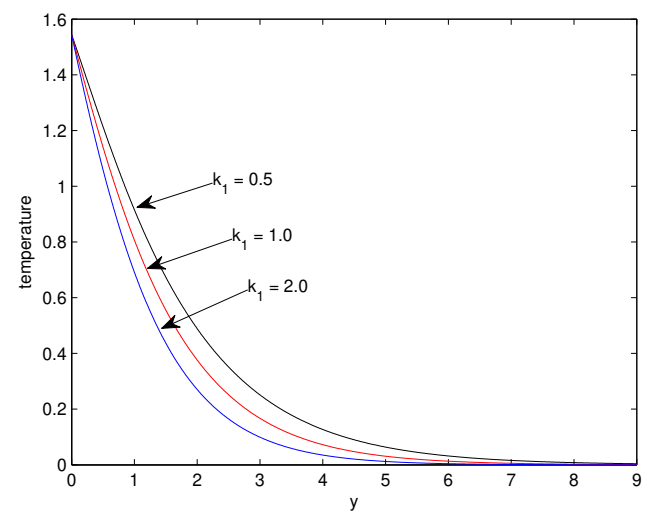

Figure 12: Temperature profile for different values of $k_{1}$

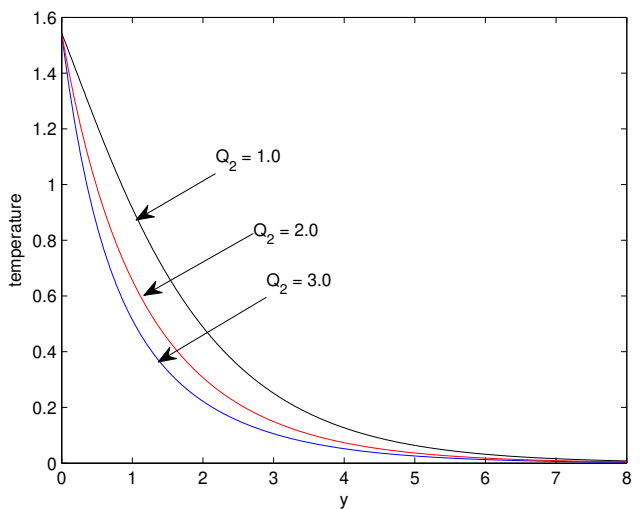

Figure 13: Temperature profile for different values of $Q_{2}$

10. Couple wall stress increases with increase in the suction parameter and magnetic parameter $M \geq 6$ and decreases with increase in the heat generation parameter and magnetic parameter $M<6$.

Most of the above findings confirms previously published work. The findings which stand out in the present study are that $C_{f}$ decreases as $k_{1}$ increases and $C_{m}$ remain unchanged as $k_{1}$ increases. $N u$ and $C_{f}$ increases as $Q_{1}$ increases while $S h$ remain unchanged. Also the Couple stress increases for magnetic parameter $M \geq 6$ and decreases otherwise. Furthermore the inclination angle has the effect of decreasing the velocity profiles and increasing the micro-rotation profiles. 


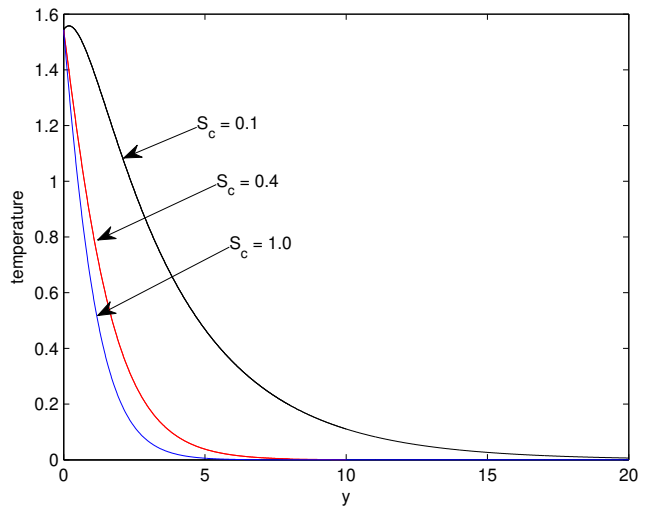

Figure 14: Temperature profile for different values of $S_{c}$

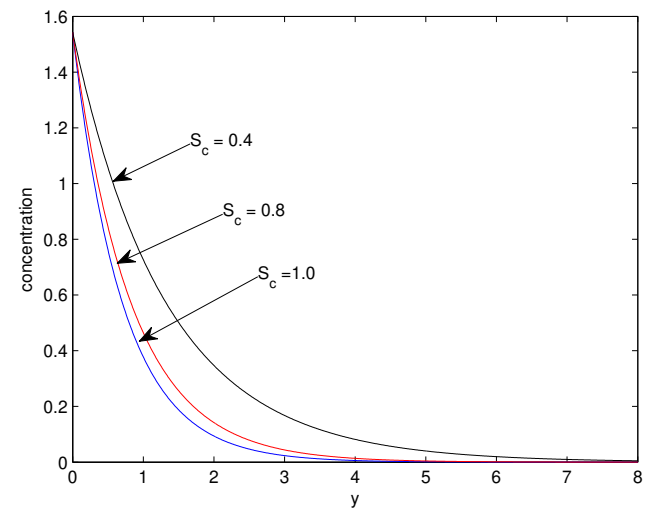

Figure 15: Concentration profile for different values of $S_{c}$

\section{References}

[1] G. Ahmadi, Self-similar solution of incompressible micropolar boundary layer flow over a semi-infinite plate, Int. J. of Engin. and Sci., 14 (1976), 639-646.

[2] M. S. Alam, M. M. Rahman and A. Satter, Effects of variable suction and thermophoresis on steady MHD combined free-forced convective heat and mass transfer flow over a semi-infinite permeable inclined plate in the presence of thermal radiation, Int. J. Therm. Sci., 47, No 6 (2008), 758-765.

[3] M. S. Alam, M. M. Rahman and A. Satter, Transient magnetohydrodynamic free 


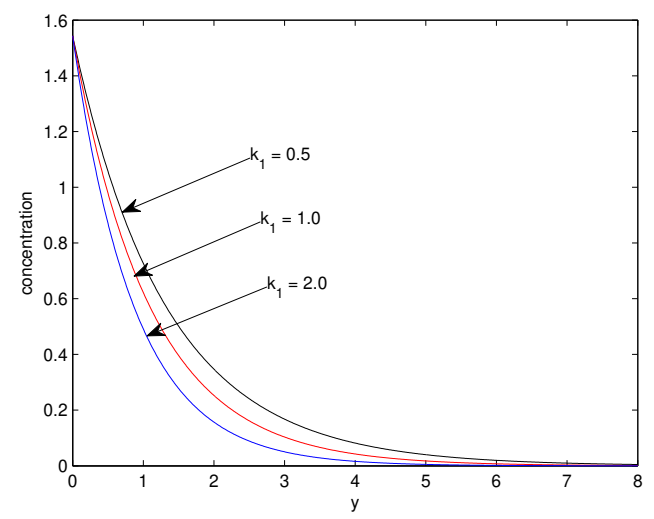

Figure 16: Concentration profile for different values of $k_{1}$

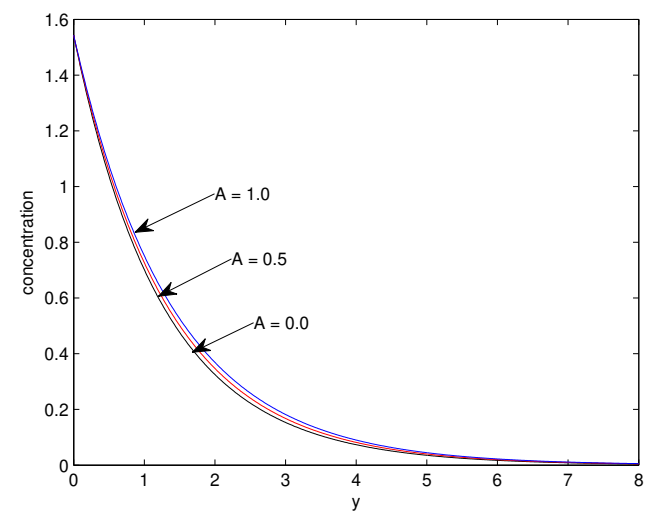

Figure 17: Concentration profile for different values of $A$

convective heat and mass transfer flow with thermophoresis past a radiate inclined permeable plate in the presence of variable chemical reaction and temperature dependent viscosity, Nonlinear Analysis: Model Control, 14 (2009), 3-20.

[4] A. A. Bakr, Effects of chemical reaction on MHD free convection and mass transfer flow of a micropolar fluid with oscillatory plate velocity and constant heat source in a rotating of reference, Commun. Nonlin. Sci. Numer. Simulat. (2010); DOI:10.1016/j.cnsns.2010.04.040.

[5] G. Buzuzi, J. B. Munyakazi and K. C. Patidar, A fitted numerical method to 
Table 2: Effect of $Q_{2}$ on skin friction, Nusselt number, Couple wall stress and Sherwood number

\begin{tabular}{ccccc}
\hline$Q_{2}$ & $C_{f}$ & $C_{m}$ & $N u R e^{-1}$ & $S h R e^{-1}$ \\
\hline 0.0 & 16.4882 & 56.4193 & 0.7223 & 0.4641 \\
0.3 & 16.2641 & 55.7937 & 0.9513 & 0.4641 \\
0.5 & 16.1673 & 55.5201 & 1.0636 & 0.4641 \\
\hline
\end{tabular}

Table 3: Effect of $A$ on skin friction, Nusselt number, Couple wall stress and Sherwood number

\begin{tabular}{ccccc}
\hline$A$ & $C_{f}$ & $C_{m}$ & $N u R e^{-1}$ & $S h R e^{-1}$ \\
\hline 0.0 & 17.3350 & -0.1245 & 58.5943 & 0.4641 \\
0.5 & 17.6373 & -0.1244 & 59.8199 & 0.4641 \\
1.0 & 17.9396 & -0.1243 & 61.0456 & 0.4633 \\
\hline
\end{tabular}

investigate the effect of various parameters on an MHD flow over an inclined plate, Numer. Methods for Partial Diff. Equations (2015), 107-120.

[6] G. Buzuzi and A. N. Buzuzi, Unsteady MHD convection flow and heat transfer past a vertical inclined plate in a porous medium with variable plate temperature with suction in a slip flow regime, Int. J. of Appl. Math., 32, No 2 (2019), 205-218; DOI: $10.12732 /$ ijam.v32i2.4.

[7] A. J. Chamkha, MHD flow of a uniformly stretched vertical permeable surface in the presence of heat generation/absorption and a chemical reaction, Int Comm. Heat Mass Transfer, 30 (2003), 413-422.

[8] R. Deka, U. N. Das, Effects of mass transfer on flow past an impulsively started infinite vertical plate with constant heat flux and chemical reaction, Forsch Ingenieurwes, 60 (1994), 284-287.

[9] A. C. Eringen, Theory of micropolar fluids, J. Math. Mech., 16 (1996), 1-18.

[10] F. M. Hady, R. A. Mohamed and A. Mahdy, MHD Free convection flow along a vertical wavy surface with heat generation or absorption effect, Int. Comm. Heat Mass Transfer, 33 (2006), 1253-1263.

[11] F. S. Ibrahim, A. M. Elaiw and A. A. Bakr, Effect of the chemical reaction and radiation absorption on the unsteady MHD free convection flow past a semi infinite vertical permeable moving plate with heat source and suction, Commun. in Nonlin. Sci. and Numer. Simul., 13 (2008), 1056-1066. 
Table 4: Effect of $Q_{1}$ on skin friction, Nusselt number, Couple wall stress and Sherwood number

\begin{tabular}{ccccc}
\hline$Q_{1}$ & $C_{f}$ & $N u R e^{-1}$ & $C_{m}$ & $S h R e^{-1}$ \\
\hline 1.0 & 16.8163 & 0.5810 & 57.4237 & 0.4641 \\
2.0 & 17.6373 & -0.1244 & 59.8199 & 0.4641 \\
3.0 & 18.4583 & -0.8299 & 62.2161 & 0.4641 \\
4.0 & 19.2793 & -1.5353 & 64.6124 & 0.4641 \\
\hline
\end{tabular}

Table 5: Effect of $k_{1}$ on skin friction, Nusselt number, Couple wall stress and Sherwood number

\begin{tabular}{ccccc}
\hline$k_{1}$ & $C_{f}$ & $N u R e^{-1}$ & $C_{m}$ & $S h R e^{-1}$ \\
\hline 0.0 & 16.4782 & 56.3016 & 1.0636 & 0.2223 \\
0.1 & 16.3716 & 56.0335 & 1.0636 & 0.2976 \\
0.5 & 16.1673 & 55.5201 & 1.0636 & 0.4641 \\
1.0 & 16.0285 & 55.1713 & 1.0636 & 0.5978 \\
2.0 & 15.8591 & 54.7455 & 1.0636 & 0.7905 \\
\hline
\end{tabular}

[12] Y. J. Kim, Unsteady MHD convection flow of polar fluids past a vertical moving porous plate in a porous medium, Int. J. of Heat and Mass Transfer, 44 (2001), 2791-2799.

[13] Y. J. Kim and G. Fedorov, Transient mixed radiative convection flow of a micropolar fluid past a moving semi-infinite vertical porous plate, Int. J. of Heat and Mass Transfer, 46 (2003), 1753-1761.

[14] S. T. Reddy, R. V. Prasad, P. Roja and B. N. Reddy, Radiation effects on MHD mixed convection flow of a micropolar fluid past a semi infinite plate in a porous medium with heat absorption, Int. J. of Appl. Math. and Mech., 6, No 18 (2010), $80-101$.

[15] P. Roja, T. S. Reddy and N. B. Reddy, Double diffusive convection-radiation interaction on unsteady Mhd flow of a micropolar fluid over a vertical moving porous plate embedded in a porous medium with heat generation and soret effects, Int. J. of Engin. and Sci., 1, No 2 (2012), 201-214.

[16] R. Sharma R, R. Bhargava and I. V. Singh, Combined effect of magnetic field and absorption on unsteady free convection and heat transfer flow in a micropolar 
Table 6: Effect of $\psi$ on skin friction, Nusselt number, Couple wall stress and Sherwood number

\begin{tabular}{ccccc}
\hline$\psi$ & $C_{f}$ & $N u R e^{-1}$ & $C_{m}$ & $S h R e^{-1}$ \\
\hline 0.0 & 32.9937 & 0.1028 & 105.2355 & 0.6095 \\
$\pi / 6$ & 30.0485 & 0.1028 & 95.7873 & 0.6095 \\
$\pi / 3$ & 22.0018 & 0.1028 & 69.9745 & 0.6095 \\
$\pi / 2$ & 11.0099 & 0.1028 & 34.7134 & 0.6095 \\
\hline
\end{tabular}

fluid past a semi-infinite moving plate with viscous dissipation using element free Garlekin method, Appl. Math. and Comput., 217 (2010), 308-321.

[17] V. M. Soundalgeker and H. S. Takhar, Flow of a micropolar fluid on a continuous moving plate, Int. J. of Engin. and Sci., 21 (1983), 961-965.

[18] M. J. Uddin, Convective flow of micropolar fluids along an inclined flat plate with variable electric conductivity and uniform surface heat flux, DAFFODIL Int. Univ. J. of Sci. and Techn., 6, No 1 (2011), 69-79. 